EXTENSION SUPERVISORS USE FOR INFORMATION AND COMMUNICATION TECHNOLOGY IN AGRICULTURAL EXTENSION WORK IN SOME DELTA GOVERNORATES El-Shafei,A.E.A.; A. M. El-zahby and R. H. A.E. Abou zaiud Agricultural Extension and Rural Development Research Institute, A.R.C.

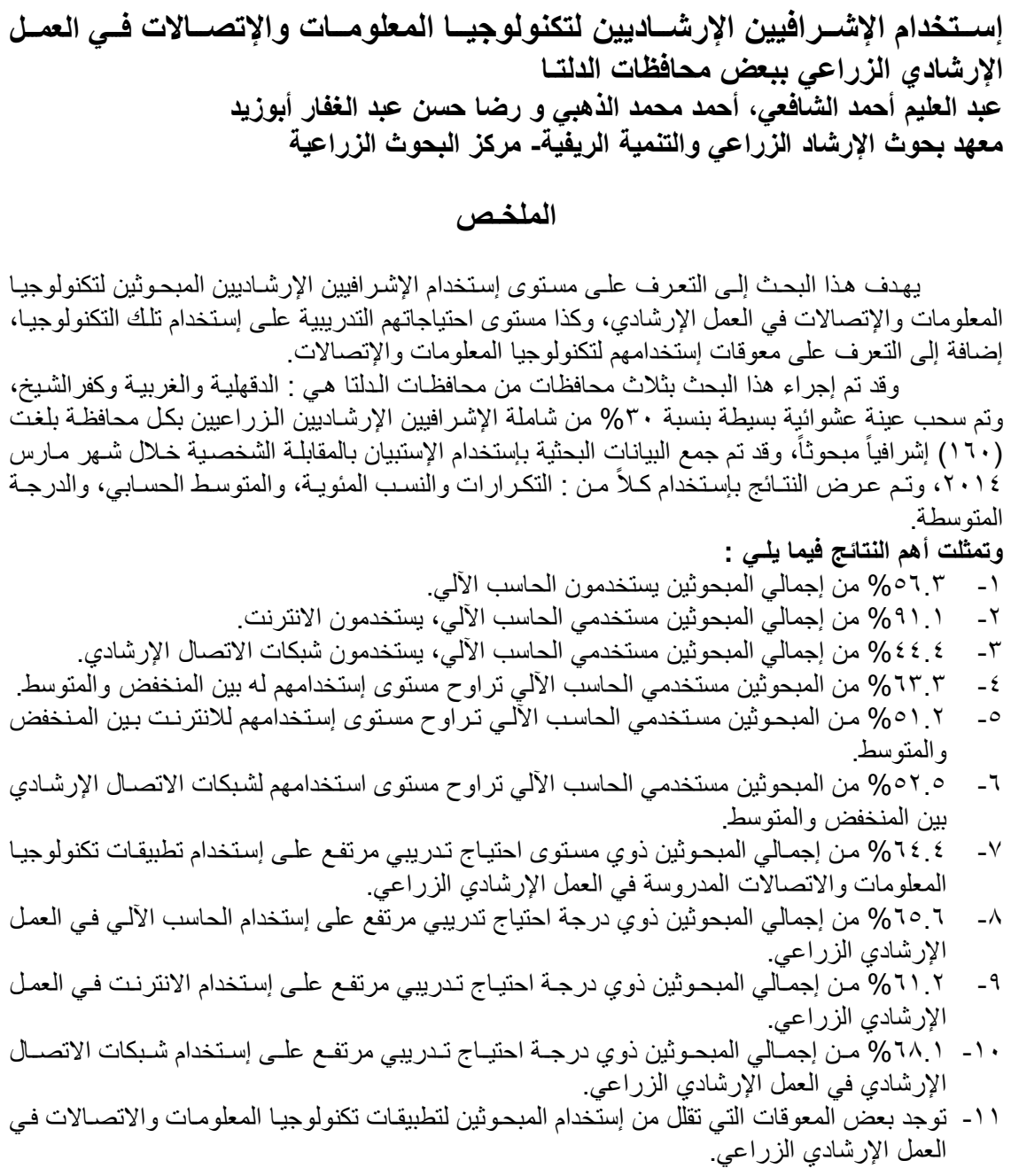




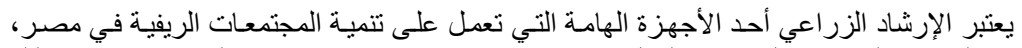

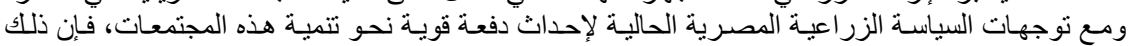

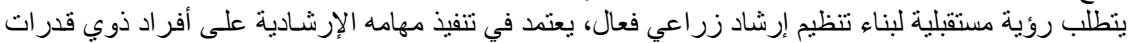
ومهار ات عالية لتحقيق دوره التنموي.

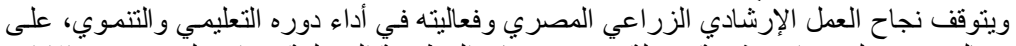

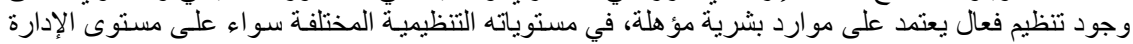

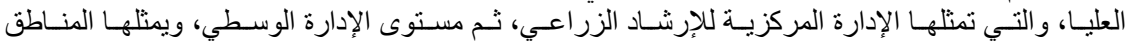

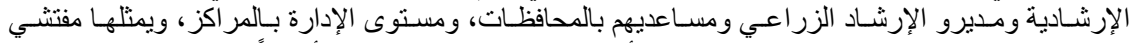

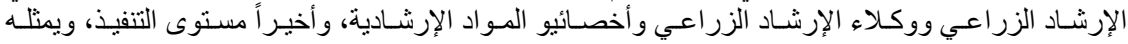

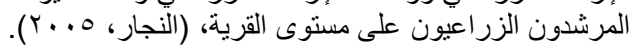

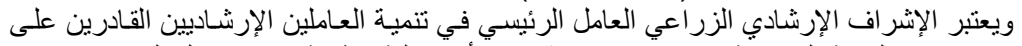

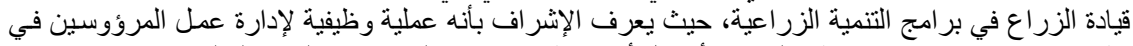

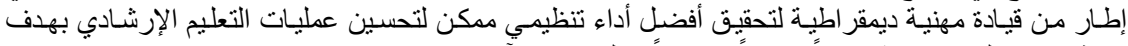

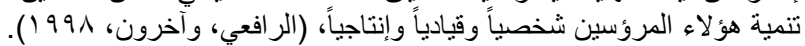

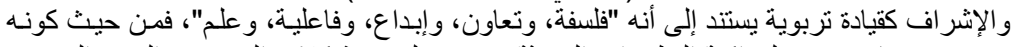

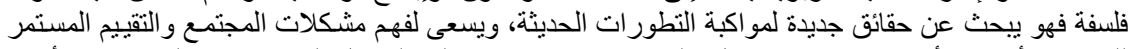

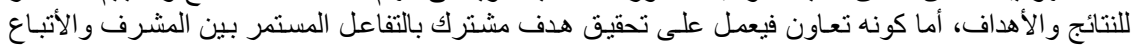

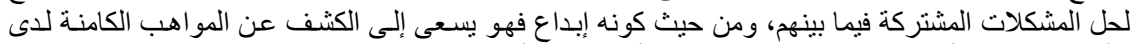

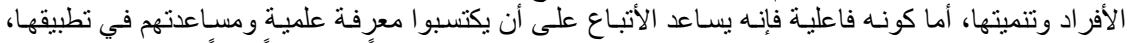

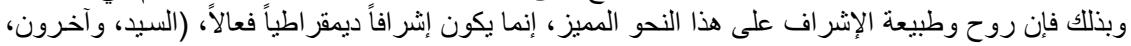

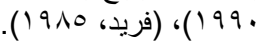

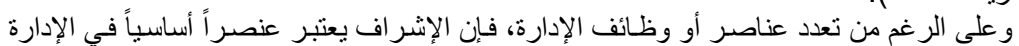

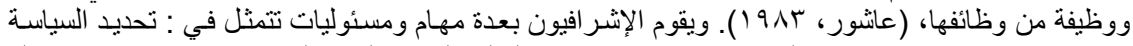

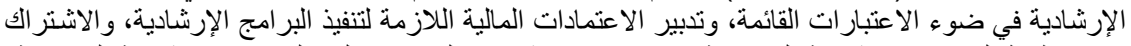

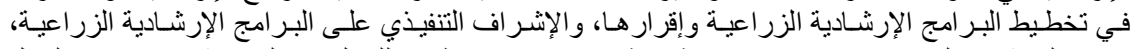

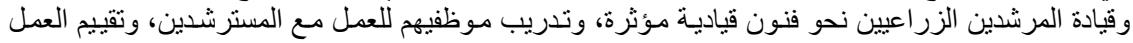

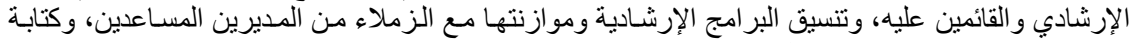

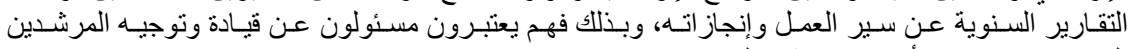

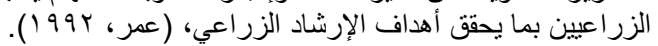

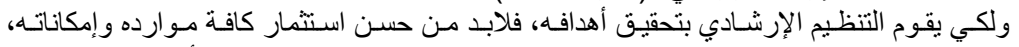

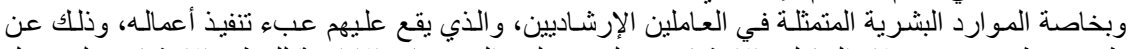

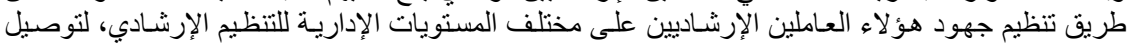

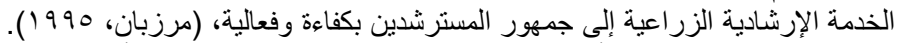

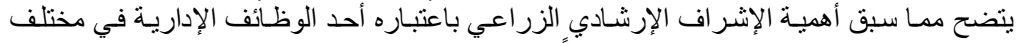

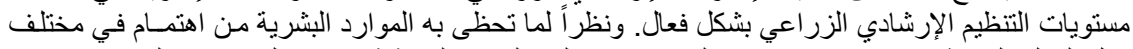

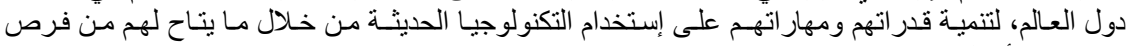

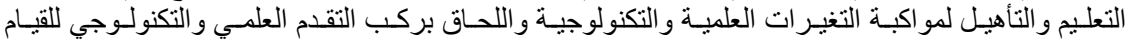

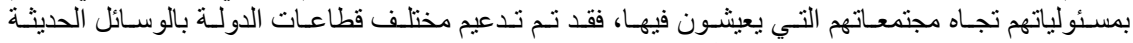

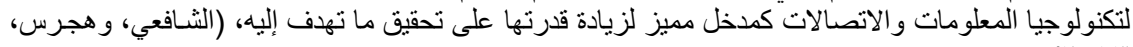

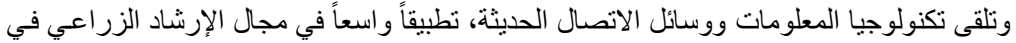

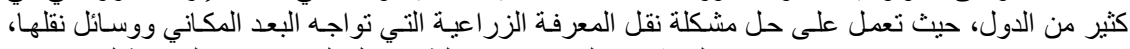

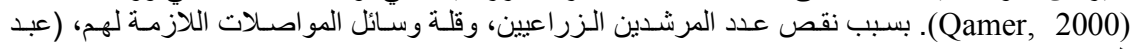

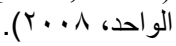

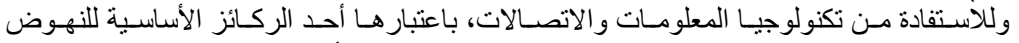

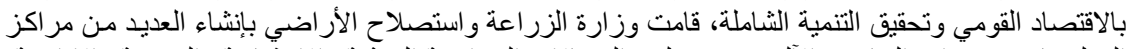

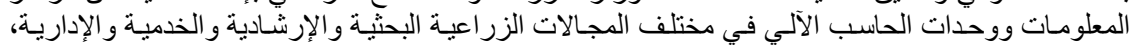


وذللك بغرض خدمة العمل في القطاع الزراعي، وضمان سر عة تدفق المعلومات الزراعية واتخـاذ القرارات

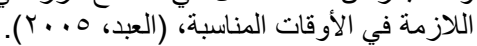

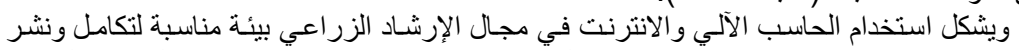

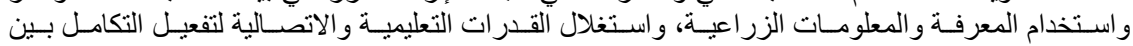

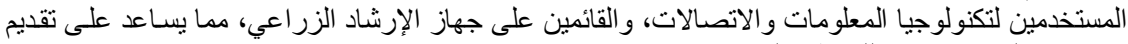

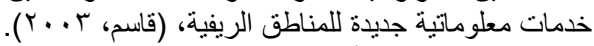

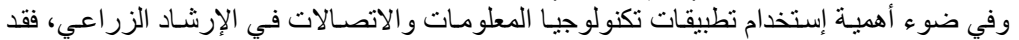

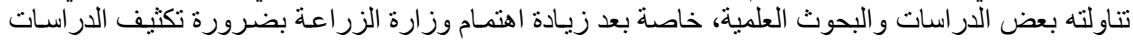

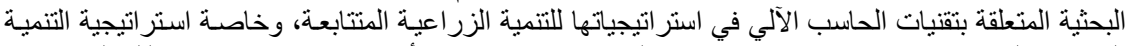

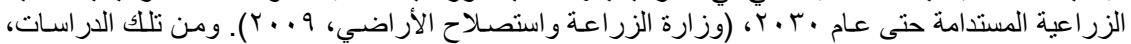

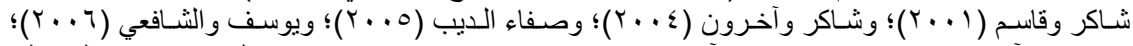

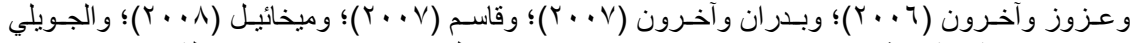

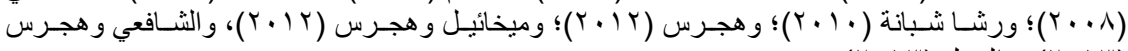

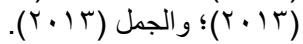

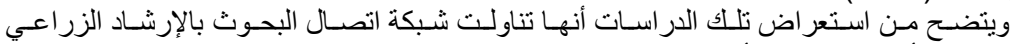

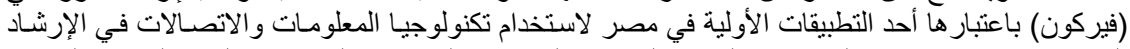

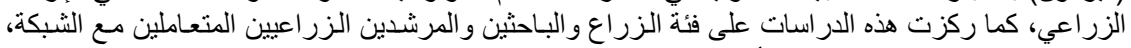

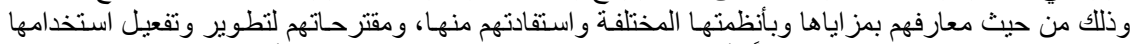

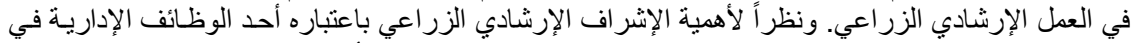

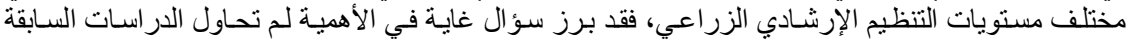

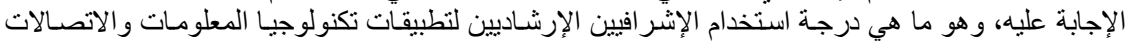

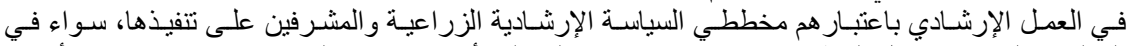

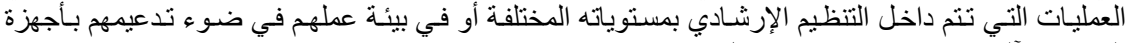

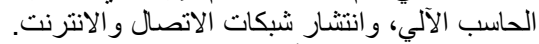

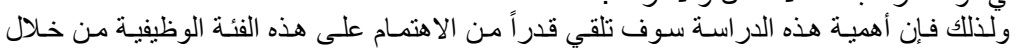

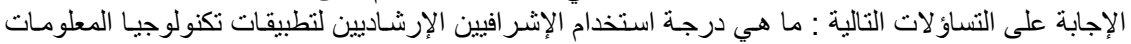

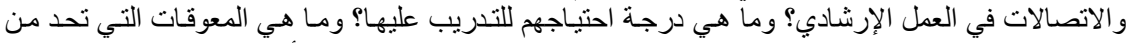

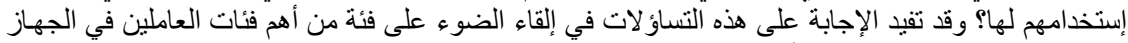

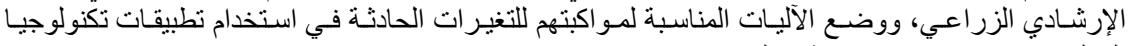

$$
\text { المعلومات و الاتصالات في بيئة عملهم. الإليات }
$$

بينتهدف البحث تحقيق الأهداف التالية :

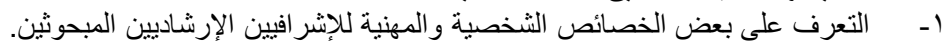

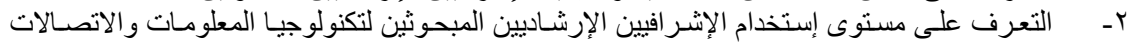

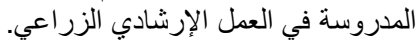

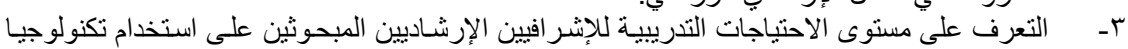

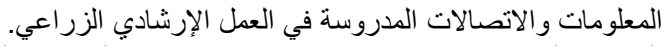

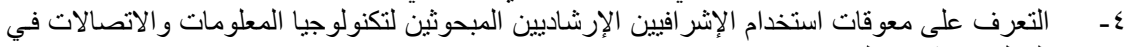
العمل الإرشادي الزرفي معات اعني.

\section{الطريقـة البحثية}

التعريفات الإجرائيـة : الإنير

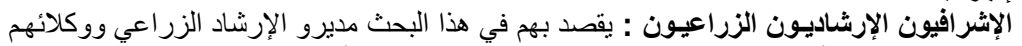

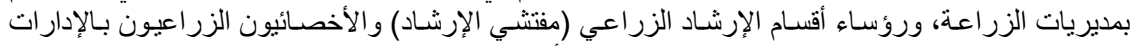

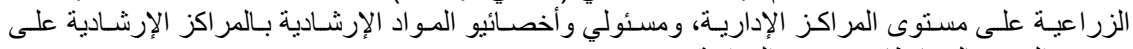
مستوى القرى بالمحافظات موضع الدر استة الإسة 


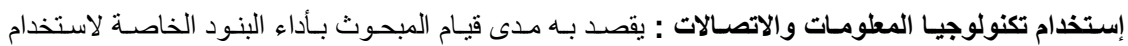

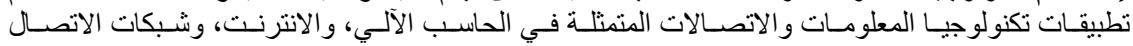

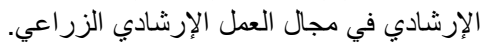

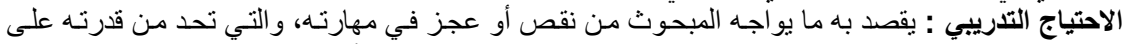

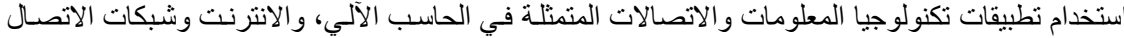

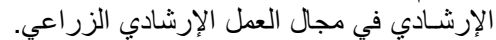

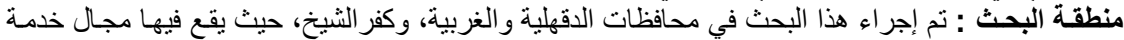

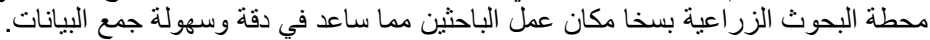

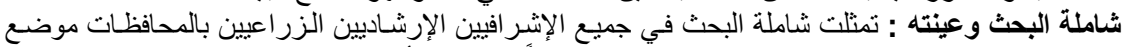

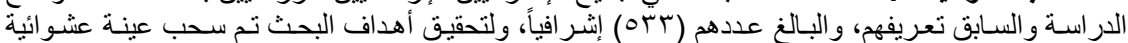

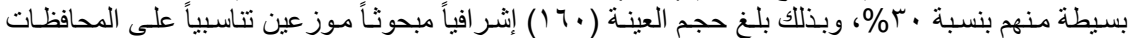
موضع الدراسة على النحو التالي، جدول ( ) : (1) :

جدول (1) : توزيع شاملة البحث وعينته على المحافظات موضع الدراسة.

\begin{tabular}{|c|c|c|c|c|}
\hline الإجمالـي & كفرالشيخ & الغربيـة & الدقهيـة & المحافظات المختارة \\
\hline OrT & $1 \wedge \varepsilon$ & 117 & $17 \pi$ & الثشاملـة \\
\hline 17. & 00 & 07 & $\leqslant 9$ & العينــة \\
\hline
\end{tabular}

أسلوب جمع البيانـات : تم الحصول على البيانات اللازمة لتحقيق أهداف البحث باستخدام الاستبيان بالمقابلة

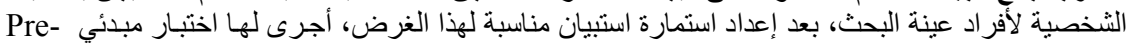

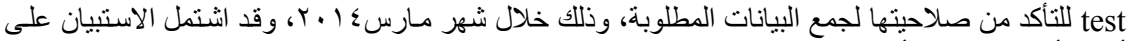

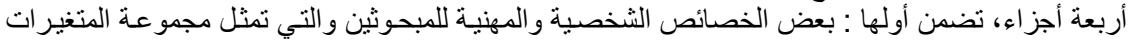

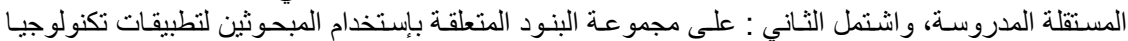

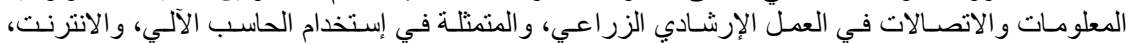

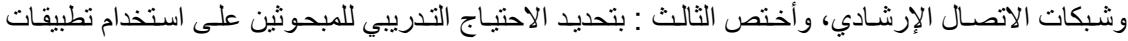

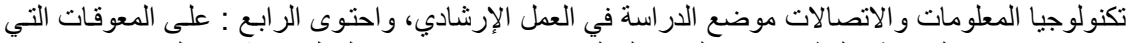

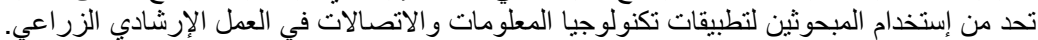

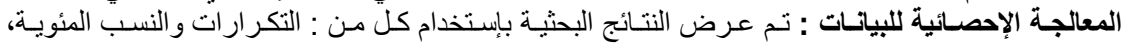

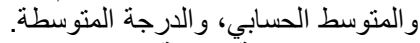
المتغيرات البحثية وكيفية قياسها : التئية

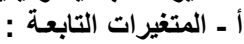

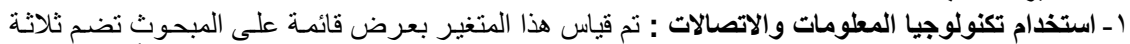

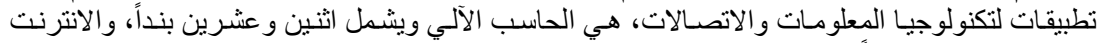

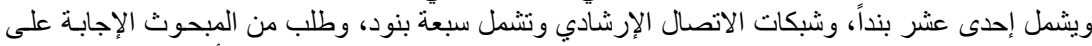

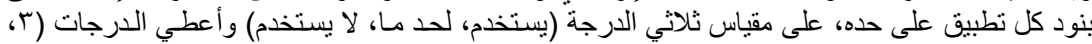

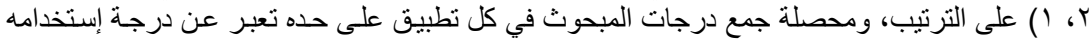

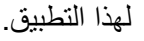

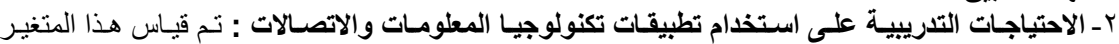

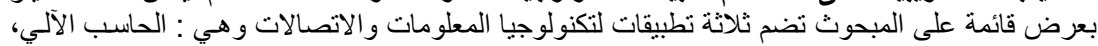

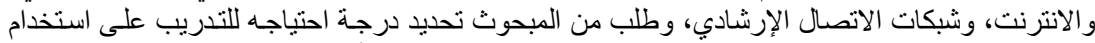

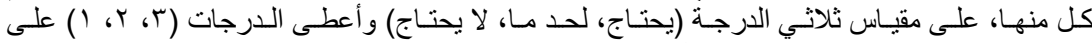

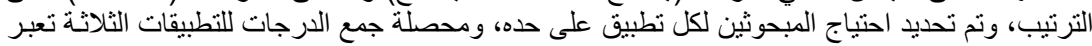

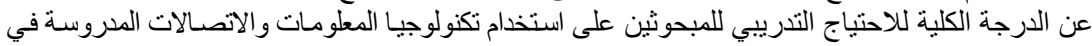

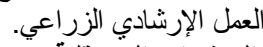

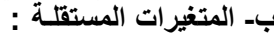
ا ـ سن المبحوث : تم قياسه بالرقم الخام لعدد سنوات عمر المبحوث وقت جمع البيانات. 


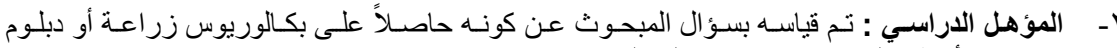

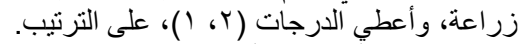

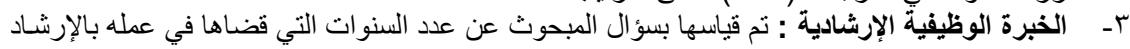
الزراعي.

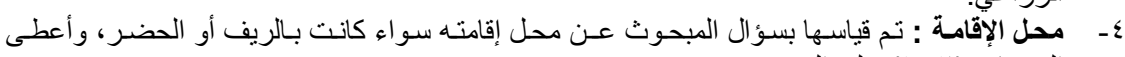

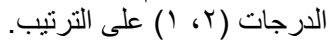

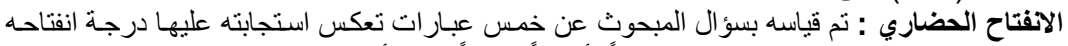

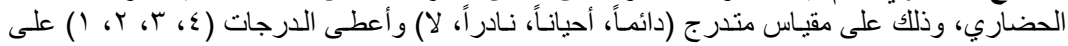

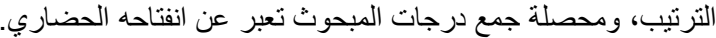

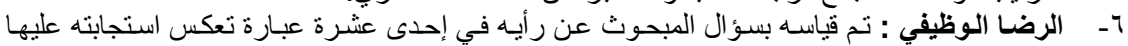

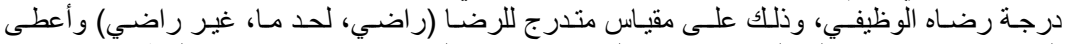

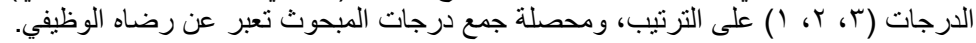

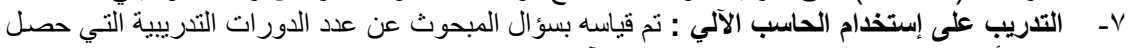

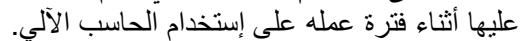

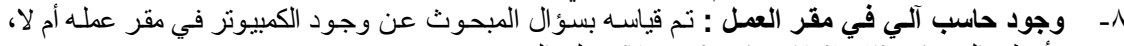

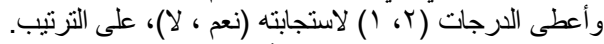

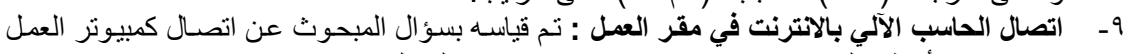

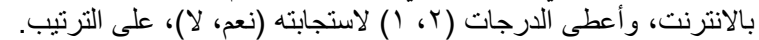

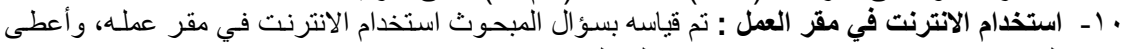

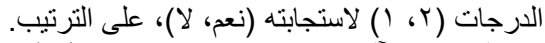

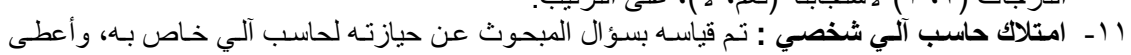

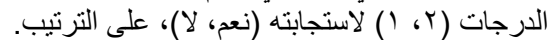

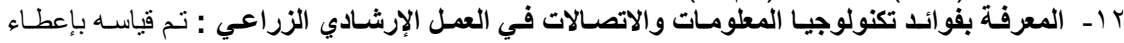

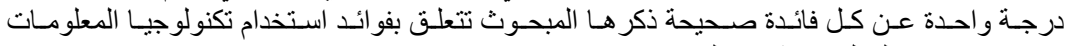
والاتصالات في العمل الإرشادي الزراعي.

\section{التتائسج ومناقشتثها}

اـ الخصائص الثخصية والمهنية للإشرافيين الإرشاديين المبحوثين :

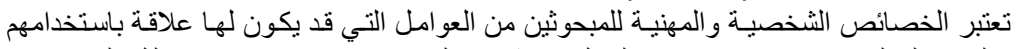

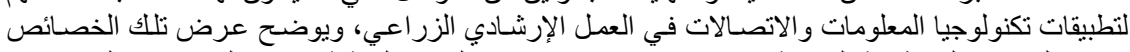

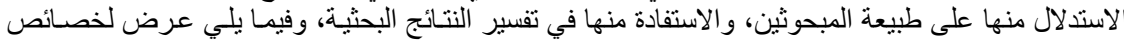

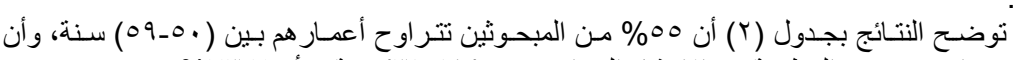

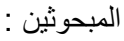

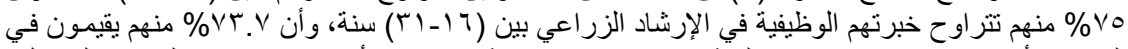

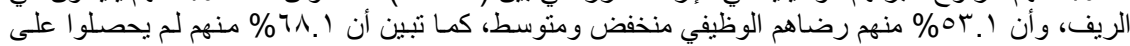

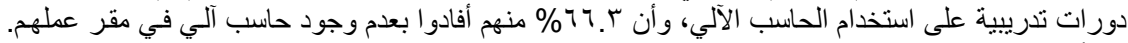

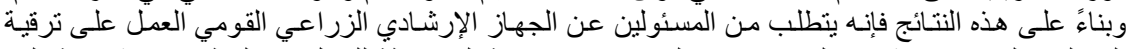

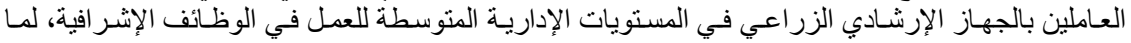

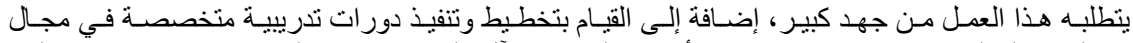

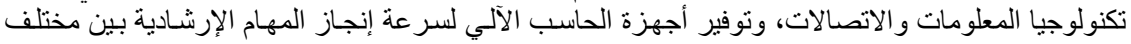

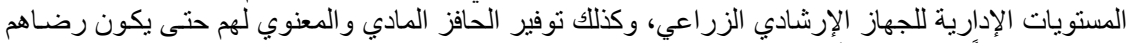

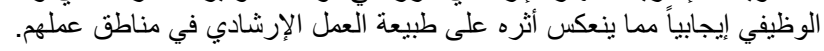


El-Shafei,A. E. A. et al.

جدول (r) : توزيع الإشرافيين الإرشاديين المبحوثين وفقاً لخصائصهم الشخصية والمهنية المدروسة.

\begin{tabular}{|c|c|c|c|c|c|c|c|}
\hline$\%$ & العدد & الخصائص والفئـات & 5 & $\%$ & العدد & الخصائص والفئـات & 5 \\
\hline & & التدريب على استخدام الحاسب الآلي & $-\mathrm{V}$ & & & | الســـن : & -1 \\
\hline 71.1 & 1.9 & لم يحصل على دورات & & $11 . r$ & 11 & |من (·•r- & \\
\hline YA.1 & $\leqslant 0$ & من (1- Y (Y) دورة & & Tr.V & $0 \leqslant$ & |من (•؟- 9 ؟) سنة & \\
\hline \multirow[t]{2}{*}{$r . \wedge$} & 7 & من (r- ع) دورة & & 00. & $\Lambda \Lambda$ & من (0.09-09) سنة & \\
\hline & & 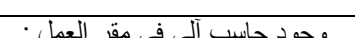 & & & & . & \\
\hline rT. & $0 \leqslant$ & ابوجد & & $v 7.9$ & IrT & |بكالو ربوس زريسية اعة & -1 \\
\hline \multirow[t]{3}{*}{$77 . r$} & 1.7 & ل لا يوجد & & rT.I & rV & 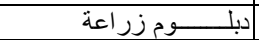 & \\
\hline & & & & & & & \\
\hline & & اتصال الحاسب بالنت في مقر العمل : & -9 & & & |الخبرة الوظيفية الإرشادية: & -4 \\
\hline $0 . . \cdot$ & trV & متصل & & ro. & $\varepsilon$. & من (^- 10 (1) سنة & \\
\hline \multirow[t]{2}{*}{$0 .}$. & trV & غير متصل & & r. & 0. & | من (T & \\
\hline & & & & $\varepsilon r . V$ & v. & 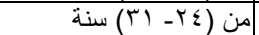 & \\
\hline \multirow[t]{2}{*}{$1 \cdots \cdot$} & $0 \leqslant$ & ن ن & & & & & \\
\hline & & إستخدام النت في مقر العمل : & $-1 \cdot$ & & & | محل الإقامــة : & $-\varepsilon$ \\
\hline$v \varepsilon .1$ & r. & يستخـــدم & & $V T . V$ & 111 & ريف & \\
\hline ro.9 & $\mathrm{v}$ & ل ل اليتخذم & & rฯ. & $\varepsilon r$ & 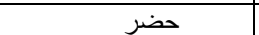 & \\
\hline \multirow[t]{2}{*}{$1 \cdots \cdot$} & rV & ن & & & & & \\
\hline & & امتلاك حاسب آلي شخصي : & -11 & & & |الانفتاح الحضـاري : & -0 \\
\hline $7 \leqslant . \varepsilon$ & 1.4 & يمتلاك & & 19.5 & $r$ & من (1- 1') درجة & \\
\hline \multirow[t]{4}{*}{ ro. 7} & ov & لا يمتلك & & $0 \cdot$. & $\wedge$. & |من (rا ـ م1) درجة & \\
\hline & & & & $r \cdot .7$ & $\leqslant 9$ & |من (7 (1 - 9 1) درجة & \\
\hline & & المعرفة بفو ائد تكنولوجيا المعلومات & $-1 Y$ & & & & \\
\hline & & و الاتصالات في العمل الإرشادي & & & & |الرضا الوظيفي : & -7 \\
\hline 17.1 & $r 7$ & لا بعـرف & & 11.1 & Y) & 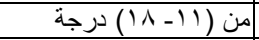 & \\
\hline$\sum 7 . r$ & $V \varepsilon$ & من (1 - Y) درجة & & $\varepsilon \cdot . \cdot$ & $7 \leq$ & |من (19- צY) درجة & \\
\hline rv.o & 7. & من (r- ع ) درجة & & $\leq 7.9$ & vo & |من (VV- Tr & \\
\hline
\end{tabular}

r- إسـتخدام الإثـرافيين الإرشــاديين المبحـوثين لتكنولوجيـا المطلومـات والاتصـالات المدروسـة في العـل

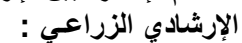

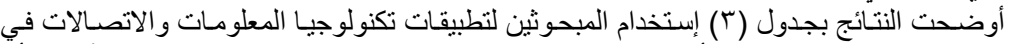

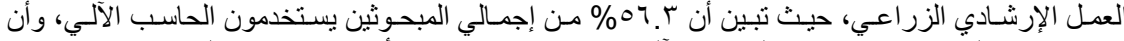

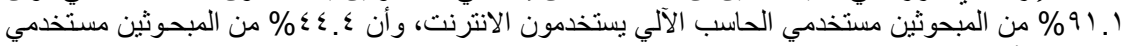
الحاسب الآلي يستخدمون شبكات الاتصال الإرشادي. 


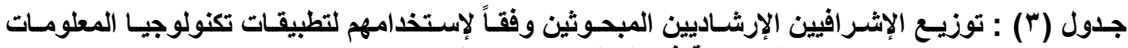

\begin{tabular}{|c|c|c|c|c|c|c|}
\hline \multicolumn{2}{|c|}{ شبكات الآتصال } & \multicolumn{2}{|c|}{ الإنتــــتت } & \multicolumn{2}{|c|}{ الحاسب الآلـــــي } & \multirow[t]{2}{*}{ المسطويقات والاتصنالات تكوليات } \\
\hline$\%$ & عـداد & $\%$ & عـداد & $\%$ & عـداد & \\
\hline$\leqslant \leqslant . \leqslant$ & $\varepsilon \cdot$ & 91.1 & AT & $07 . r$ & 9. & بيستخـدم \\
\hline 00.7 & 0. & $\Lambda .9$ & $\Lambda$ & $\varepsilon r . Y$ & $V \cdot$ & لا بستخذم \\
\hline $1 \ldots$ & 9. & $1 \ldots$ & 9. & $1 \ldots$ & 17. & الإجمالـح \\
\hline
\end{tabular}

ولمزيد مـن الإيضـاح يمكن عرض النتائج التي تثـير إلى مستوى استخدام المبحوثين لتطبيقات

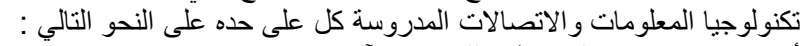

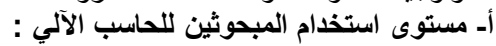

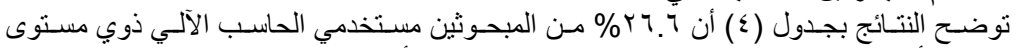

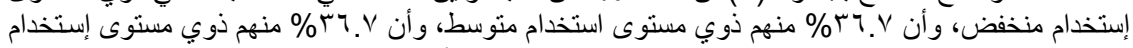

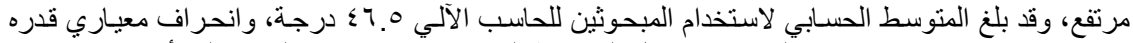

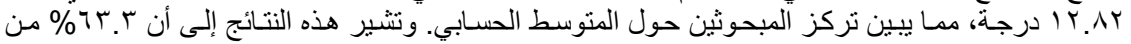

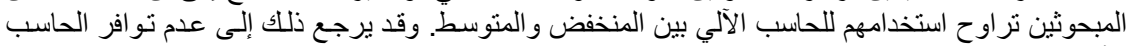
الآلي في مقر عمل المبحوثين، وقلة عقد دورات تدريبية لهم بالقدر الكافي في هذا لئن المجال.

جدول (ء) : توزيع الإشرافيين الإرشـاديين المبحوثين وفقاً لمستوى إستخدامهم للحاسب الآلي في العمل

\begin{tabular}{|c|c|c|}
\hline$\%$ & العـدد & فئات مستوى الإستخدام \\
\hline YY. & $r \leq$ & منخفض (r \\
\hline r..V & rr & منوسط (rV- \\
\hline r..V & rr & 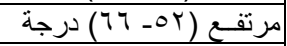 \\
\hline $1 \ldots$ & 9. & الإجمال \\
\hline
\end{tabular}

ب- مستوى استخدام المبحوثين للانترنت :

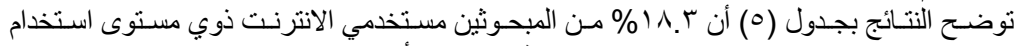

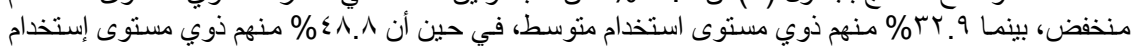

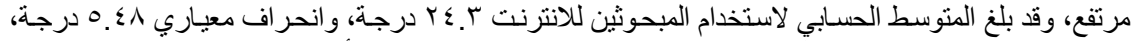

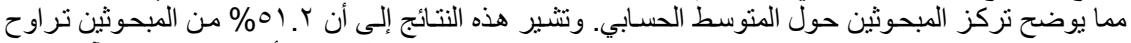

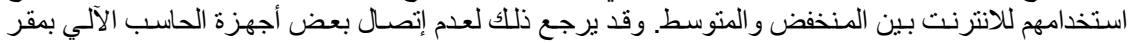
عملهم بشبكة الانترنت.

جدول (0) : توزيع الإشرافيين الإرشاديين المبحوثين وفقاً لمستوى استخدامهم للانترنت في العمل الإرشـادي

\begin{tabular}{|c|c|c|}
\hline$\%$ & العـدد & فئات مستوى الإستخذام \\
\hline $1 \wedge . r$ & 10 & منخفض (1 - 1 |) درجة \\
\hline rY.q & TV & متوسط (9 (- 7Y) درجة \\
\hline$\sum \wedge . \wedge$ & $\varepsilon$. & مرتفـع (YV- YYT) درجة \\
\hline $1 \ldots$ & NY & الإجمالـ \\
\hline
\end{tabular}

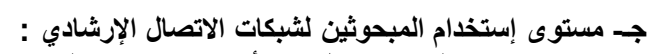

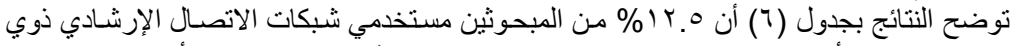

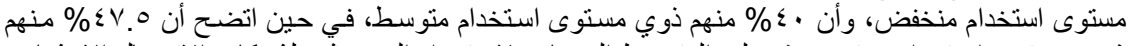

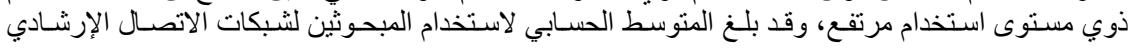




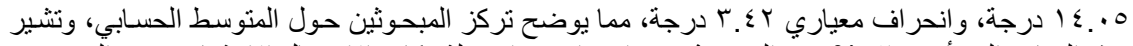

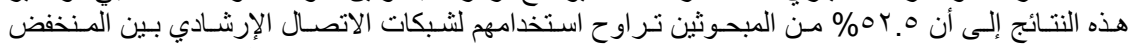

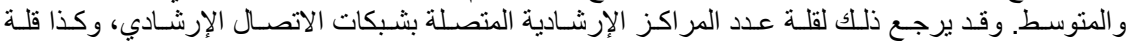
الأخصائيين الإرشاديين المسئولين عن إدارة تلألك الثبكات.

جدول (†) : توزيع الإشرافيين الإرشاديين المبحوثين وفقائاً لمستوى استخدامهم لشبكات الاتصـال الإرشـادي في العمل الإرشادي الزراعي.

\begin{tabular}{|c|c|c|}
\hline$\%$ & العـدد & قفئات مستوى الاستخدام \\
\hline Ir.O & 0 & منخفض (V - I I) درجة \\
\hline$\varepsilon \cdot$. & 17 & متوسط (r (- 7 1) درجة \\
\hline$\sum \vee .0$ & 19 & مرتفـع (V) - (Y) درجة \\
\hline $1 \ldots$ & $\varepsilon$. & الإجمالــــي \\
\hline
\end{tabular}

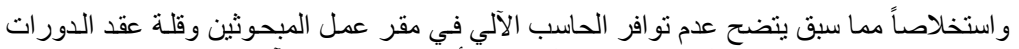

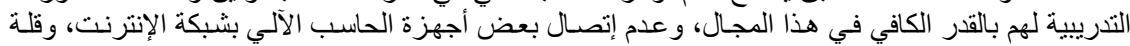

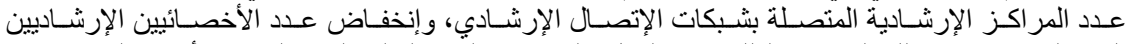

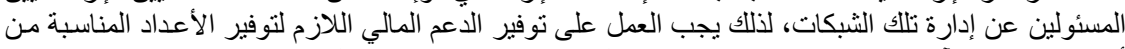

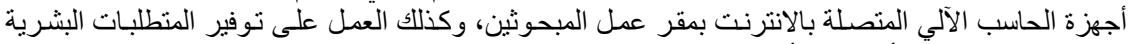

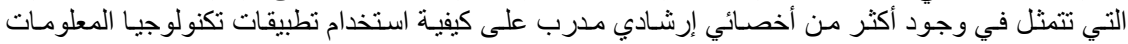

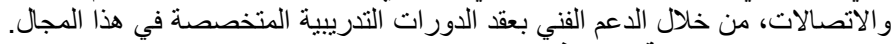

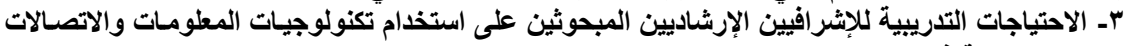

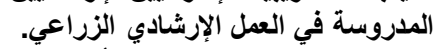

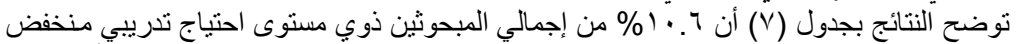

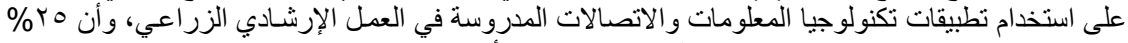

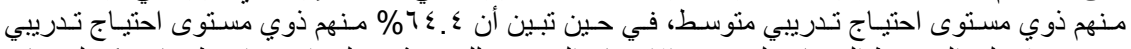

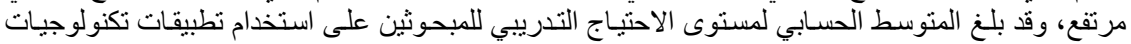

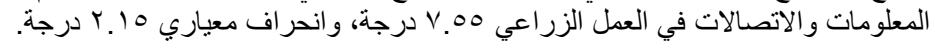

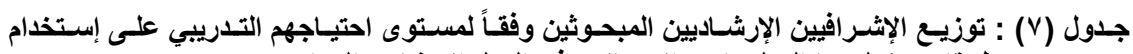

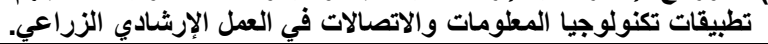

\begin{tabular}{|c|c|c|}
\hline$\%$ & العــد & فئات مستوى الإستخدام \\
\hline $1 \cdot .7$ & IV & منخفض (ع - 0) درجة \\
\hline TO. & $\varepsilon$ & متوسط (T- V) درجة \\
\hline $7 \varepsilon . \Sigma$ & $1 . r$ & مرتفـع (^- 9) درجة \\
\hline $1 \ldots$ & 17. & الإجمالــــــي \\
\hline
\end{tabular}

ولمزيد من الإيضاح يمكن عرض النتائج التي تشير إلى درجـة الاحتياج التدريبي للمبحوثين على الإنى

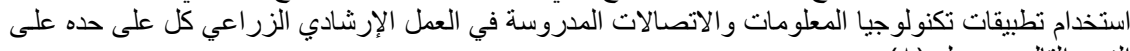

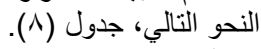
جدول (^) : توزيع الإشرافيين الإرشاديين المبحوثين وفقاً لارجة احتياجهم التدريبي على استخدام تطبيقات

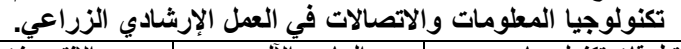

\begin{tabular}{|c|c|c|c|c|c|c|}
\hline \multicolumn{2}{|c|}{ شبكات الاتصال الإرشـــادي } & \multicolumn{2}{|c|}{ الإنتـــرنت } & \multicolumn{2}{|c|}{ الحاسب الآلـــي } & \multirow{2}{*}{ 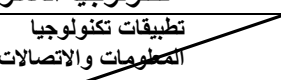 } \\
\hline$\%$ & عـد & $\%$ & عـدد & $\%$ & عـدد & \\
\hline$\Lambda . \wedge$ & $1 \leqslant$ & $11 . r$ & in & 9.9 & 11 & منخفض (1) درجة \\
\hline rY.1 & $r v$ & rV.O & $\varepsilon$ & $r V_{0}{ }^{\prime}$ & $\varepsilon \varepsilon$ & متوسط (Y) درجة \\
\hline 71.1 & 1.9 & 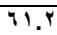 & 91 & 70.7 & 1.0 & مرتفع (ץ) درجة \\
\hline $1 \ldots$ & 17. & $1 \ldots$ & 17. & 1.. & 17. & 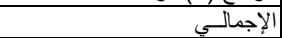 \\
\hline
\end{tabular}




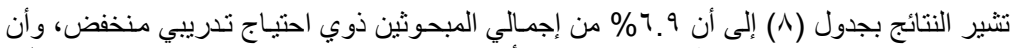

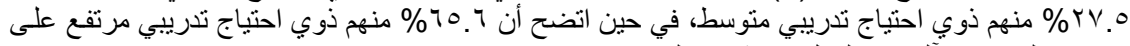

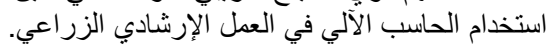

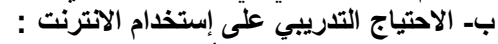

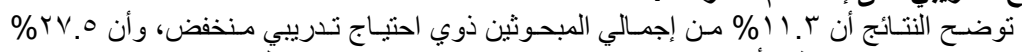

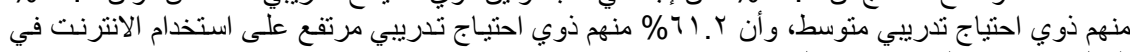

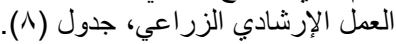

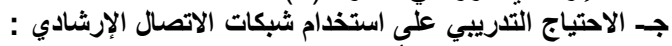

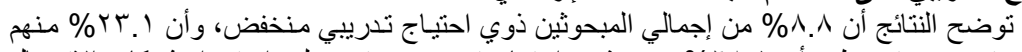

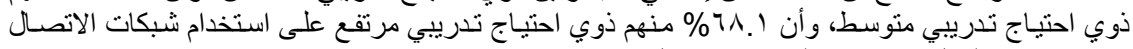

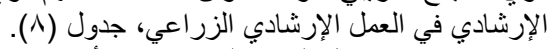

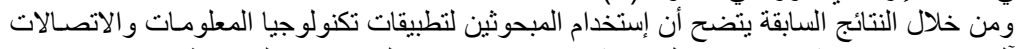

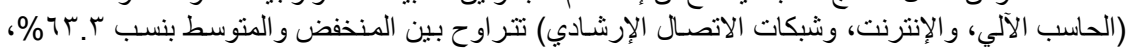

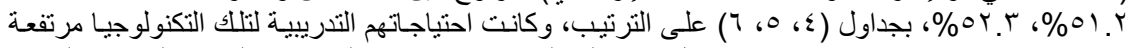

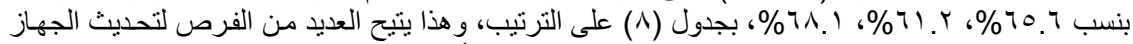

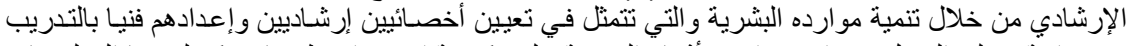

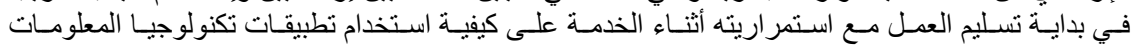

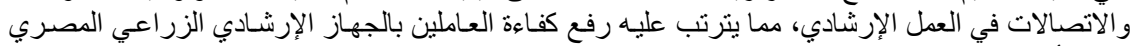

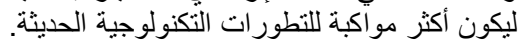

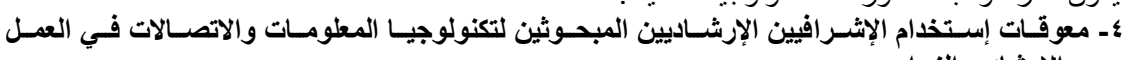

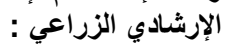

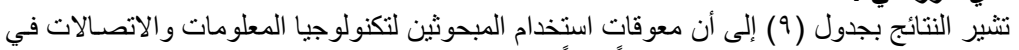

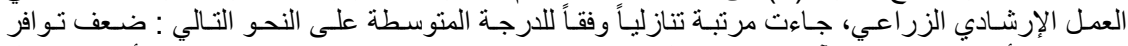

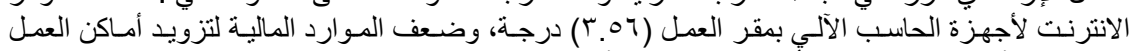

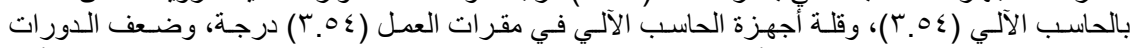

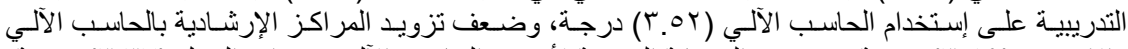

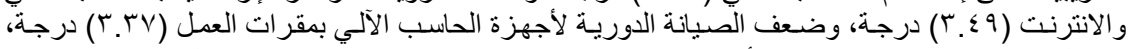

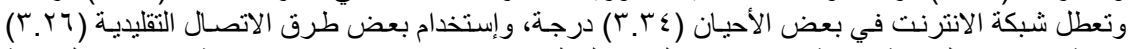

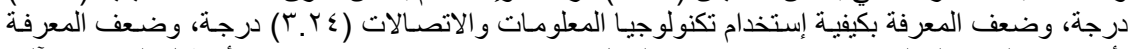

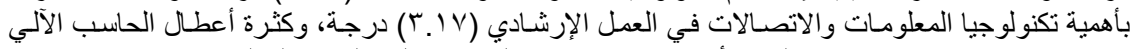

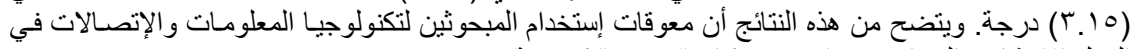

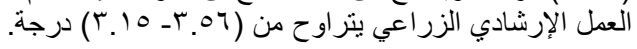

جدول (9) : ترتيب معوقات إستخدام الإشرافيين الإرشاديين المبحوثين لتكنولوجيا المطلومات والإتصالات

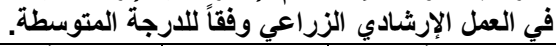

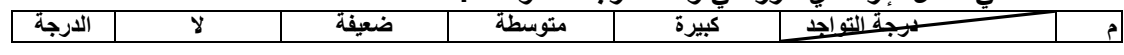


El-Shafei,A. E. A. et al.

\begin{tabular}{|c|c|c|c|c|c|c|c|c|c|c|}
\hline المتوسطة & $\%$ & عدد & $\%$ & عدد & $\%$ & عدد & $\%$ & عدد & 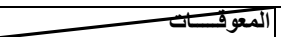 & \\
\hline 5.07 & 0. & $\wedge$ & $9 . \varepsilon$ & 10 & $1 \cdot$. & 17 & vo. 7 & $|r|$ & |الآلى بمقر العز الاتترنت لأجهزة الحاسب & 1 \\
\hline T.0 & $7 . r$ & 1. & ^. $\vee$ & $1 \varepsilon$ & $1 \cdot$. & 17 & vo. & IT. & | العمل بالحاسب الآلي المالية لتزويد أمـاكن & Y \\
\hline T.0ะ & r.V & 7 & $1 \cdot$. & 17 & $1 \leqslant . \varepsilon$ & Tr & v1.9 & 110 & | قلحة أجهزة الحاسب الآلي في مقرات & $r$ \\
\hline T.OT & $\varepsilon . \varepsilon$ & V & $1 \cdot .7$ & iv & IT.V & rr & $v i r$ & $11 \leq$ & 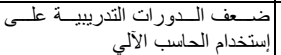 & $\varepsilon$ \\
\hline r. $\leqslant 9$ & $\varepsilon . \varepsilon$ & V & A.V & $1 \leq$ & $r \cdot .7$ & 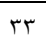 & $77 . r$ & 1.7 & 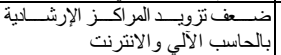 & 0 \\
\hline r.rv & ^.) & $1 \pi$ & $1 \cdot .7$ & IV & 17.9 & TV & $7 \varepsilon . \varepsilon$ & $1 . r$ & 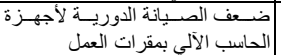 & 7 \\
\hline r.Tร & 0.7 & 9 & $1 Y .0$ & $r$. & $r \leqslant . \varepsilon$ & rq & ov.o & 94 & 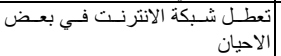 & V \\
\hline T.Y & $1 \cdot \cdot$ & 17 & A.V & $1 \leq$ & Y..9 & $\varepsilon r$ & $0 \leqslant . \varepsilon$ & AV & 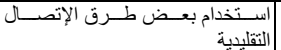 & $\wedge$ \\
\hline T.Y & $1 \cdot .7$ & iv & $11 . r$ & 11 & r. & $r \leq$ & 07.9 & 91 & 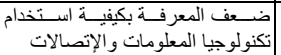 & 9 \\
\hline T.IV & $11 . r$ & 11 & $1 \cdot$. & 17 & rq. $\varepsilon$ & $\varepsilon V$ & rq. & $\vee q$ & 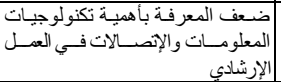 & 1. \\
\hline 5.10 & $1 \cdot .7$ & IV & $1 \leqslant . \varepsilon$ & rt & $Y \leqslant . \varepsilon$ & ra & 0.7 & $\wedge 1$ & كثَرة أعطـال الحاسب الآلي & 11 \\
\hline
\end{tabular}

في ضوء مـا أسفرت عنـه النتائج البحثية، تم استخلاص مجموعة من التوصيات تمثل الأهمية التطبيقية

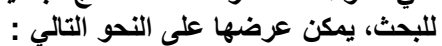

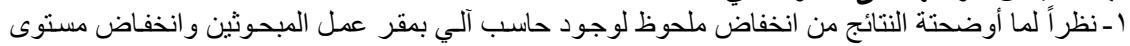

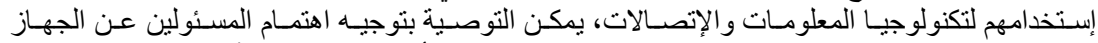

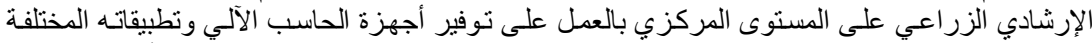

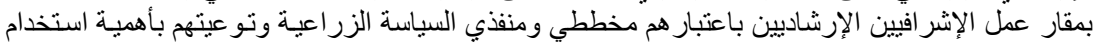
هذه التطبيقات لإنجاز مهامهم الإرشادية.

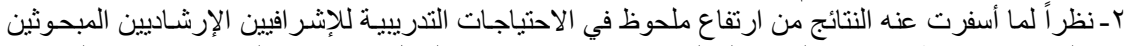

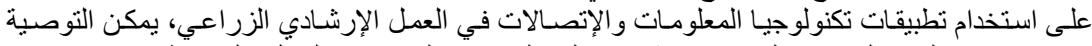

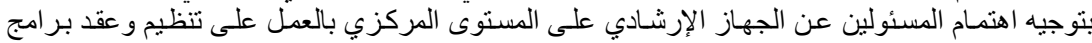

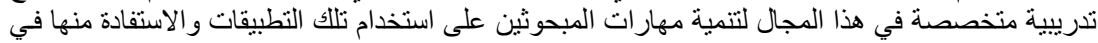

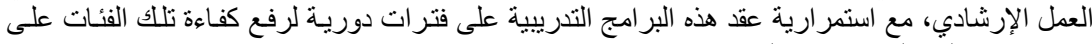

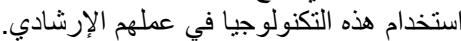

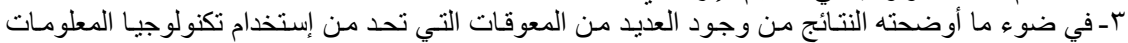

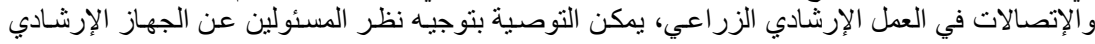

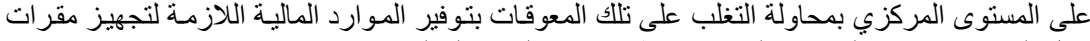

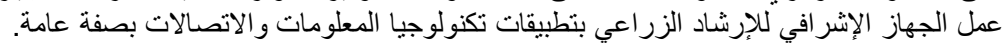

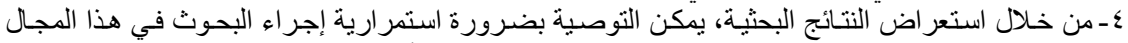

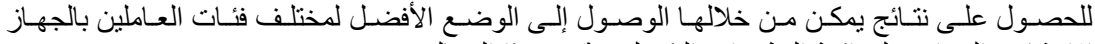

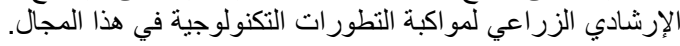




\section{المراجـع}

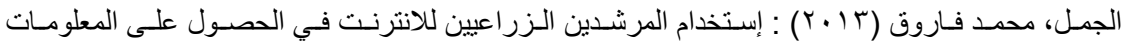

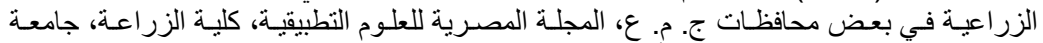

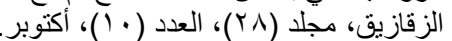

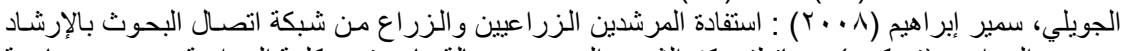

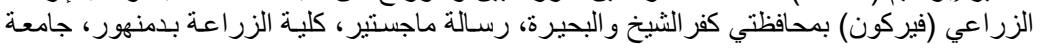
الإسكندرية.

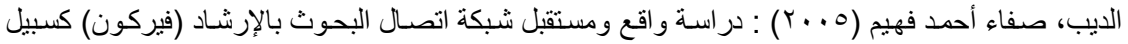

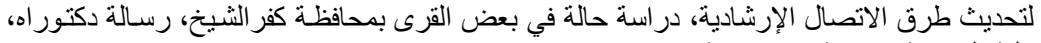
كلية الزر اعة، جامعة الإسكندرية.

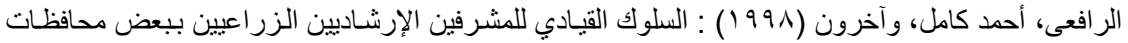

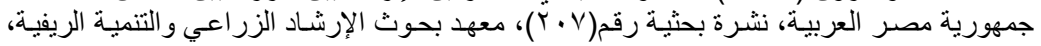
مركز البحوث الزراعية.

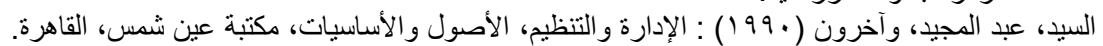

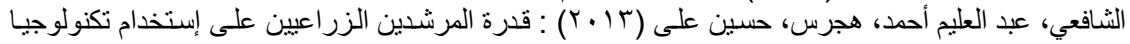

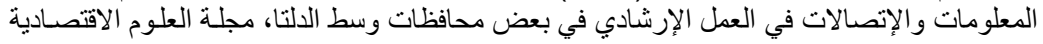

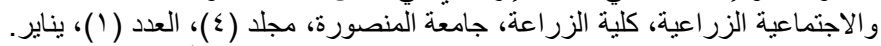

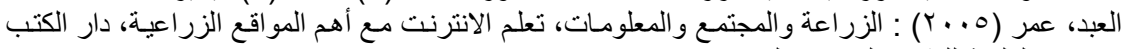

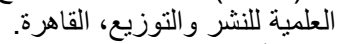

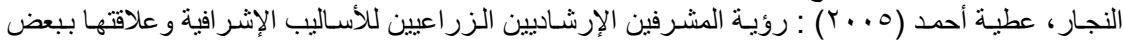

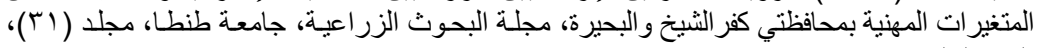

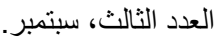

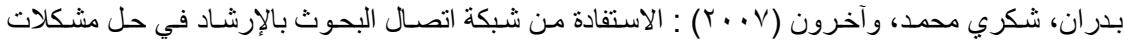

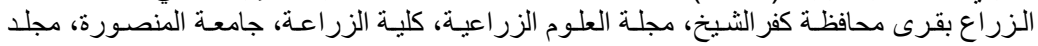

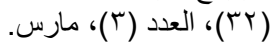

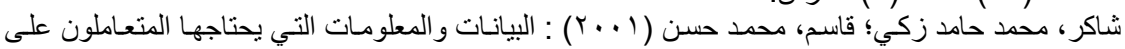

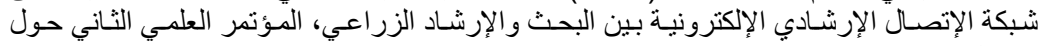

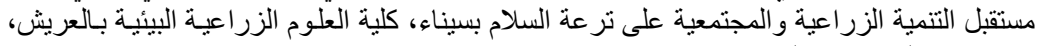

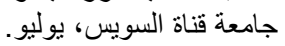

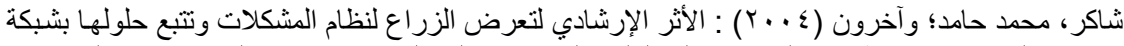

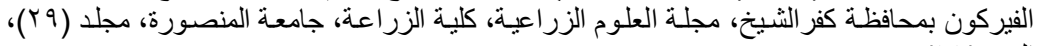

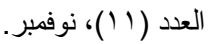

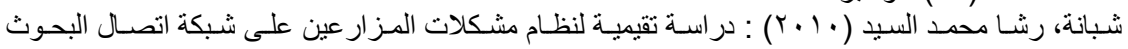

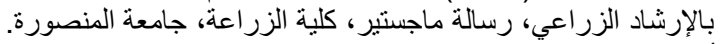

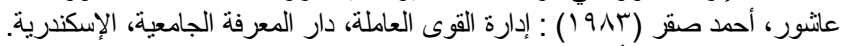

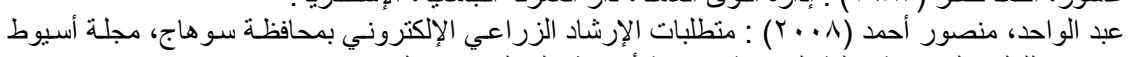

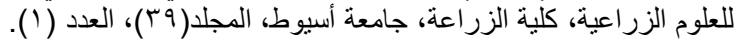

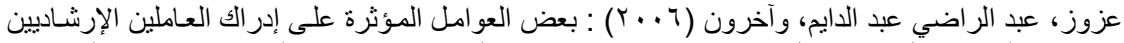

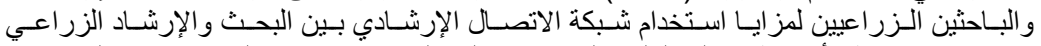

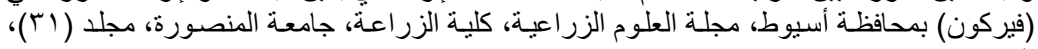

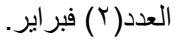

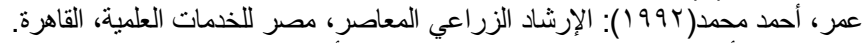

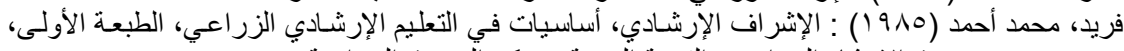

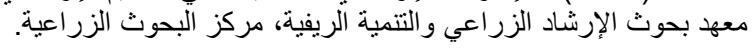




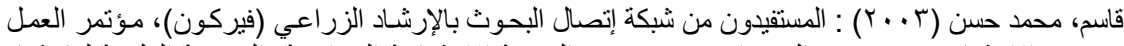

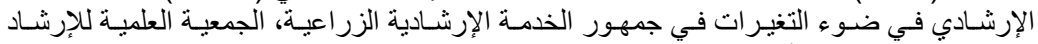

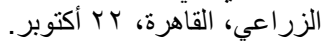

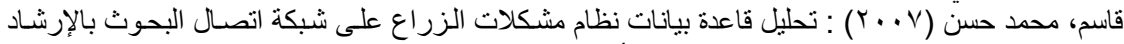

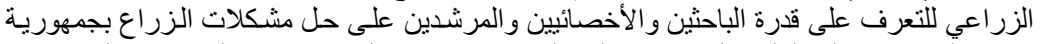

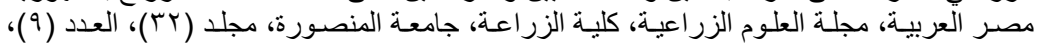
سبتمبر.

مرزبـان، عبد الحليم أحمد خلف (1990) : منهجيـة العمل الإرشـادي للجهـاز الإنشرافي الإرشـادي، دليل

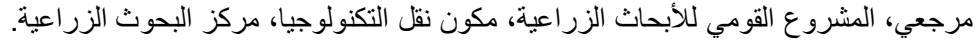

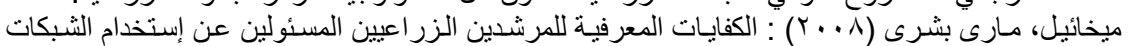

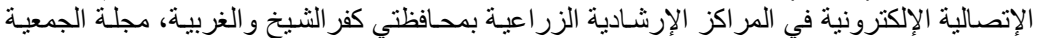

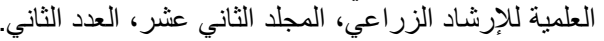

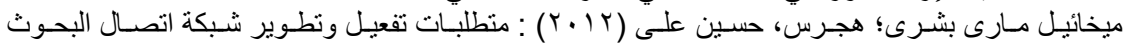

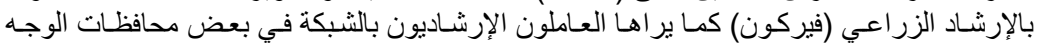

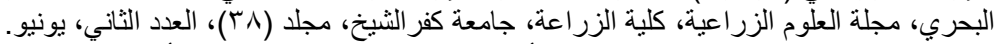

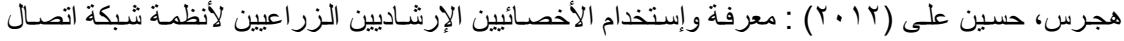

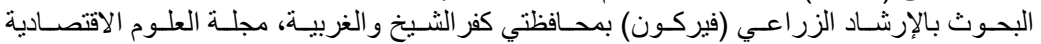

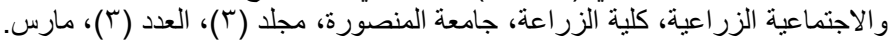

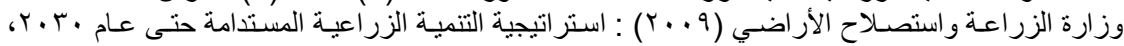
مركز البحوث الزراعية، الجيزة.

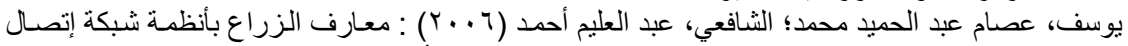

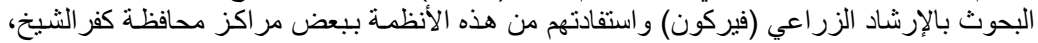

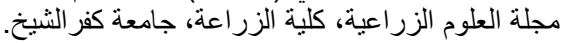

Qamer, M.K., (2000): Agricultural Extension, at the turn of Millennin, trends Challenges, F.A.O., Rome. 


\title{
EXTENSION SUPERVISORS USE FOR INFORMATION AND COMMUNICATION TECHNOLOGY IN AGRICULTURAL EXTENSION WORK IN SOME DELTA GOVERNORATES El-Shafei,A.E.A.; A. M. El-zahby and R. H. A.E. Abou zaiud Agricultural Extension and Rural Development Research Institute, A.R.C.
}

\begin{abstract}
This research aims to identify the level of use of extension supervisors respondents for information and communication technology in extension work, as well as the level of their training needs on the use of this technology, in addition to identification of obstacles to their use of information and communication technology.

The research was conducted in three governorates are: Dakahlia, Gharbia and Kafrelshaikh, they were selected randomly. Simple random sample represent $30 \%$ was selected of the population of extension supervisors in each governorate. The sample amounted to (160) respondents, research data has been collected by using a personal interview questionnaire during the month of March 2014, data were analyzed and results are presented using both: frequencies, percentages, the arithmetic mean, and the medium degree.

The most important results were as follows:

$1-56.3 \%$ of the total respondents use computer.

$2-91.1 \%$ of the total respondents, who are users of computer use internet.

$3-44.4 \%$ of the total respondents, who are users of computer use extension communication networks.

$4-63.3 \%$ of the respondents who are users of Computer, their level of using it was ranged between low and medium.

$5-51.2 \%$ of the respondents who are users of Computer, their level of using internet was ranged between low and medium.

$6-52.5 \%$ of the respondents who are users of Computer, their level of using extension communication networks was ranged between low and medium.

$7-64.4 \%$ of the total respondents have high level of need for training in the use of applications of the studied information and communication technology in agricultural extension work.

$8-65.6 \%$ of the total respondents have high degree of need for training in the use of computer in agricultural extension work.

$9-61.2 \%$ of the total respondents have high degree of need for training in the use of internet in agricultural extension work.

$10-68.1 \%$ of the total respondents have high degree of need for training in the use of extension communication networks in agricultural extension work.

11-There are some obstacles which reduce the respondents use for the applications of information and communication technology in agricultural extension work.
\end{abstract}


EI-Shafei,A. E. A. et al.

$\begin{array}{llllllllllll}1480 & 1481 & 1482 & 1483 & 1484 & 1485 & 1486 & 1487 & 1488 & 1489 & 1490 & 1491\end{array}$

$\begin{array}{llllllllllll}1480 & 1481 & 1482 & 1483 & 1484 & 1485 & 1486 & 1487 & 1488 & 1489 & 1490 & 1491\end{array}$ 